\title{
La formación del profesorado de educación secundaria en Italia y en España
}

\author{
Secondary School Teachers' Training in Italy and Spain
}

\author{
Ornella D’Aita, Ingrid Mosquera Gende \\ Universidad de Catania, Universidad Internacional de la Rioja
}

\begin{abstract}
Resumen
Mediante las últimas reformas educativas llevadas a cabo, y con cualquier reforma educativa en general, se pretende adecuar los sistemas de enseñanza a los requerimientos de la sociedad, adaptándolos, además, a la normativa europea, y asegurando, igualmente, la consecución de una buena formación del profesorado, para un óptimo aprendizaje de los discentes. Este trabajo se centrará en ese último aspecto mencionado, la formación del profesorado, desde un punto de vista comparativo, estableciendo un paralelismo entre la formación y criterios necesarios para ejercer como profesor de educación secundaria en España y en Italia. Palabras clave: formación, profesorado, educación secundaria.
\end{abstract}

\begin{abstract}
The latest educational reforms, and any educational reform in general, seek to adapt educational systems to the requirements of society, acknowledging the European legislation as well as ensuring an outstanding teacher training system in order to implement an optimal learning process for the students. This work will focus on the aspect of teacher training, comparing the necessary criteria and studies to become a secondary school teacher in Spain and Italy.

Keywords: training, teachers, secondary education.
\end{abstract}

\section{Introducción}

En esta comunicación, se intenta introducir brevemente el tema de la formación de profesorado de secundaria desde una perspectiva comparativa, considerando la formación y los criterios necesarios para ejercer como profesor de educación secundaria en España y en Italia, a partir de la presentación de los sistemas educativos en ambos países.

De hecho, a pesar del Espacio Europeo de Educación Superior (EEES), no se han homologado los requisitos y el camino es distinto, creando, así, disparidad entre los países europeos. Es necesario considerar de qué diferencias se trata y, a partir de estas, ofrecer posibles propuestas de mejora, teniendo en cuenta que, además del marco teórico presentado sobre el tema, se necesitaría un estudio de campo sobre los factores implicados.

\section{El sistema educativo en España}

El sistema educativo actual en España está definido por la Ley Orgánica de Educación (LOE) (Ley Orgánica de Educación 2/2006, 2006: p. 8). Con la Ley Orgánica de Mejora de la Calidad Educativa (LOMCE) (Ley Orgánica de Mejora de la Calidad Educativa 8/2013, 2013: p. 10), se introducen cambios relevantes. De hecho, la LOMCE no establece una nueva ley de educación propiamente dicha, sino que modifica la LOE.

En el título Preliminar de la LOE (Ley Orgánica de Educación 2/2006, 2006: pp. 7-8) se hace referencia a los principios y los fines de la educación. Entre los principios fundamentales se destaca "la calidad de la educación para todo el alumnado", "la transmisión de valores y puesta en práctica de valores que favorezcan la libertad personal, la responsabilidad, la ciudadanía democrática", "la concepción de la educación como un aprendizaje permanente”, "la participación de la comunidad educativa”. Entre los fines de la educación se señalan "el pleno desarrollo de la personalidad y de las capacidades", "la prevención de conflictos" y "la interculturalidad”.

En el título I (Ley Orgánica de Educación 2/2006, 2006: p. 10), se establece la Organización de las enseñanzas y sus etapas. El sistema educativo se estructura a través de las siguientes enseñanzas: Educación Infantil, Primaria, y Secundaria Obligatoria (ESO), Bachillerato, Formación Profesional, Enseñanzas de Idiomas, Artísticas, y Deportivas, Educación de Personas Adultas, Enseñanzas Universitarias. En comparación con la Ley Orgánica General del Sistema Educativo (LOGSE) (Ley Orgánica de Ordenación General del Sistema Educativo 1, 1990: p. 4), en este apartado hay novedades importantes, entre las cuales se destacan algunas, sin considerar las que hacen referencia al currículo, que no son, por cierto, menos interesantes. En Educación Infantil se vuelve a definir la etapa desde los 0-6 años y en Primaria se da más entidad propia a la etapa. También en Educación Secundaria se pone una atención particular a la orientación educativa y profesional y, en Bachillerato, en centros pequeños, se da la posibilidad de que los alumnos puedan cursar materias en otros institutos, añadiendo, además, una definición más precisa de la formación profesional.

Por último, cabe señalar que, en el título II de la Ley (Ley Orgánica de Educación 2/2006, 2006: p. 22), se garantiza la atención a la diversidad de todos los discentes y, en el título III (Ley Orgánica de Educación 2/2006, 2006: p. 26), se presta una atención prioritaria a la formación del profesorado, que se analizará posteriormente.

Con la LOMCE, los principales cambios son que la Educación Primaria consta ahora de seis cursos independientes y desaparecen los ciclos, y la ESO se organiza en dos ciclos, el primero de tres cursos escolares y el segundo de uno, cuarto de la ESO, con dos posibles vías, una académica y otra profesional, una encaminada a 
continuar los estudios universitarios y la otra pensada para aquellos que piensen realizar grados de formación profesional. En esta etapa de secundaria aparecen, igualmente, modificaciones en la atención a la diversidad, con programas de mejora del aprendizaje y rendimiento, así como cambios en la evaluación, con la inclusión de los estándares de aprendizaje evaluables. Además, en la LOMCE, se revitaliza la opción del aprendizaje profesional $\mathrm{y}$, por eso, se crean nuevas modalidades de formación que se desdoblan en la Formación Profesional Básica y en la Formación Profesional dual, con el fin de armonizar los procesos de enseñanza-aprendizaje entre los centros educativos y los de trabajo.

Siguiendo la página del Ministerio de Educación, Cultura y Deporte (s.f.), se explica que los sistemas educativos deben buscar el talento de cada alumno, su potencial, y no únicamente conseguir unos conocimientos básicos, que en muchas ocasiones no se alcanzan (con una tasa de abandono escolar temprano de un $25 \%$ aproximadamente), haciendo referencia a una educación personalizada. Para ello, se continúa en la página, es necesario incrementar el nivel de esfuerzo y exigencia, algo que se pretende lograr mediante la implantación de evaluaciones externas estandarizadas. Para Tiana Ferrer (2012: párr. 6), “desde el punto de vista de la función social de la educación, la LOE subraya los principios de equidad, inclusión, cohesión social y ejercicio de la ciudadanía democrática, mientras que la LOMCE enfatiza la competitividad, la movilidad social, la integración y la empleabilidad, sin mencionar siquiera la equidad”. Aunque quizás, si se considera que la LOMCE modifica o añade a la anterior, podrían sumarse todos esos aspectos mencionados.

\section{El sistema educativo en Italia}

El sistema educativo italiano se compone de tres ciclos: la Educación Primaria, que dura cinco años; la Educación Secundaria, que se compone de un primer ciclo de tres años (que corresponde a la ESO del sistema educativo español) y de un segundo ciclo de cinco años, y que se desdobla en los "Licei”, los institutos técnicos y los profesionales; la Educación Superior, que tiene tres opciones, que son la Universidad, la Formación artística, musical y de danza y los Institutos técnicos superiores regulados por las regiones. En Italia, desde la promulgación de la “Legge 296/06” (Legge 296/2006, 2006), la educación obligatoria dura diez años, hasta los dieciséis.

A partir de la “Legge 53/03” (Legge 53/2003, 2003), se realiza una reorganización del sistema educativo italiano. La Educación Infantil, Primaria y el primer ciclo de la Educación Secundaria se definen en el "Decreto del Presidente della Repubblica 89/2009” (Decreto del Presidente della Repubblica 89/2009, 2009). El segundo ciclo de la Educación Secundaria y la Educación Superior se regulan con la "Riforma Gelmini”. Con esta denominación, se hace referencia a una serie de leyes educativas promulgadas entre el 2008 y el 2010, entre las cuales se encuentra la “Legge 169/08” (Legge 169/2008,
2008), que intenta reorganizar el sistema educativo italiano.

Una diferencia sustancial entre el sistema educativo español y el italiano está representada por las modalidades de acceso a la Universidad. En el primer sistema, anterior a la LOMCE, el ingreso estaba regulado por Pruebas de acceso a Estudios universitarios oficiales de Grado (PAEG). También el alumno podía elegir su carrera universitaria sólo en función de la nota obtenida en las pruebas cuya estructura se establecía en el Real Decreto 1892/2008 (Real Decreto 1892/2008, 2008: p. 2). De acuerdo con la LOMCE, en el artículo 1.31 que modifica el artículo 38 de la LOE, (Ley Orgánica de Mejora de la Calidad Educativa 8/2013, 2013: p. 29), se establece que las Universidades podrán admitir a los discentes que hayan obtenido el título de Bachiller "exclusivamente por el criterio de la calificación final obtenida en el Bachillerato”, y “de forma excepcional” mediante "evaluaciones específicas de conocimientos y/o de competencias”. Además, "La ponderación de la calificación final obtenida en el Bachillerato deberá tener un valor, como mínimo, del $60 \%$ del resultado final del procedimiento de admisión” (Ley Orgánica de Mejora de la Calidad Educativa 8/2013, 2013: p. 29).

Esta selectividad no está en el sistema educativo italiano, con excepción de las profesiones sanitarias. Cada universidad puede decidir libremente los criterios que regulan el acceso. Esto significa que cualquier alumno puede acceder a la universidad procedente de cualquier escuela y con cualquiera nota.

Actualmente, el sistema educativo italiano es objeto de un amplio proceso de reestructuración, en el que opera, en particular, un principio reformista basado en la subsidiariedad, es decir, la descentralización administrativa que fortalece la autonomía pedagógica y organizativa de las instituciones.

\section{La formación de profesorado de secundaria en España}

En la última década, el sistema educativo español ha sufrido muchos cambios debido a las trasformaciones sociales y a la necesidad de homologarse con el contexto internacional.

Con el nacimiento del Espacio Europeo de Educación Superior (EEES), se ha implantado un Máster específico para la formación de profesorado de secundaria, con una duración de 60 créditos. Su fundamento legal, además de lo establecido en la LOE (Ley Orgánica de Educación 2/2006, 2006: p. 27), se encuentra en el Real Decreto 1393/2007 (Real Decreto 1393/2007, 2007: p. 9). Éste establece la ordenación de las enseñanzas universitarias oficiales y se deduce que, el modelo español, a diferencia de otros países, no diferencia la formación del profesorado de educación secundaria Obligatoria y Bachillerato. También, el artículo 17 (Real Decreto 1393/2007, 2007: p. 10) menciona los requisitos específicos y criterios de valoración de méritos establecidos para que los estudiantes sean admitidos al Máster. Además de lo previsto en el Real Decreto 1393/2007 (Real Decreto 1393/2007, 2007: p. 10), en la Orden del Ministerio de Educación y Ciencia 
ECI/3858/2007 (Orden del Ministerio de Educación y Ciencia ECI/3858, 2007: pp. 1-2), se establecen los requisitos de los planes de estudio, exigibles para ser profesor de secundaria, para la verificación de los títulos por parte de las universidades, y se señalan las competencias generales y específicas que deben adquirir los estudiantes que cursen el Máster.

Conforme a las directrices y exigencias de la Orden ECI/3858/2007 (Orden del Ministerio de Educación y Ciencia ECI/3858, 2007: pp. 1-2), el objetivo principal del Máster es proporcionar al futuro profesor la adquisición de una formación especializada que le habilite para el ejercicio de la enseñanza en la Educación Secundaria Obligatoria y Bachillerato, Formación Profesional y Escuelas Oficiales de Idiomas. Sin embargo, este título no es suficiente para ingresar en el Cuerpo de Profesores de Enseñanza Secundaria. Los requisitos generales y específicos siguientes para participar a los procedimientos selectivos están recogidos en los artículos 12 y 13 del Real Decreto 276/2007 (Real Decreto 276/2007, 2007: pp. 5-6).

\section{La formación de profesorado de secundaria en Italia}

En Italia, el camino para enseñar en las escuelas secundarias consiste en tener una licenciatura de cuatro o cinco años y en seguir un curso de formación de profesorado, llamado "Tirocinio Formativo Attivo" (TFA). Éste se regula en el "DM 249/2010" (Decreto Ministeriale 249/2010, 2010) e intenta fortalecer los conocimientos y desarrollar las habilidades didácticas, psicopedagógicas, organizacionales, interpersonales y comunicativas de los futuros docentes. El TFA tiene plazas limitadas y, conforme al "DM 11 novembre 2011" (Decreto Ministeriale 11 novembre 2011, 2011), hay que pasar el acceso mediante tres pruebas, entre las cuales, una está definida por el Ministerio de Educación y las otras dos por la Universidad elegida por el estudiante.

Con el cambio de legislatura, se han aportado modificaciones al "DM 249/2010” (Decreto Ministeriale 249/2010, 2010) y se han instituido cursos de formación, llamados "Percorsi Abilitanti Speciali” (PAS), para los profesionales que ya hubiesen enseñado por lo menos ciento ochenta días durante tres años escolares, aunque no tuviesen el título de formación. Los PAS presentan el mismo currículo del TFA, pero excluyen la parte dedicada a la práctica, dado que los que los siguen ya han enseñado y, sobre todo, no incluye pruebas de acceso. De hecho, estos cursos de formación han permitido regularizar la posición de los docentes interinos que habían enseñado sin el título, ya que la legislación europea, con directiva “1999/70/CEE” (Direttiva 1999/70/CE, 1999) en la cláusula 5, expresa que no puede haber contratos a tiempo determinado de más de treinta y seis meses. Además, con “DM 353/14” (Decreto Ministeriale 353/2014, 2014), se establece un sistema de puntuación diferenciada entre los que atienden los PAS y los que siguen el TFA. De hecho, cada profesor tiene una puntuación personal que se exhibe en las listas para llegar a ser titular.
Como se ha visto, en Italia, sin el título es posible enseñar, sin embargo, para ser profesor titular no es suficiente con tener la formación de profesorado, TFA o PAS, sino que es necesario aprobar una oposición. La última se convocó en 2012, con "DDG 82 del 24 settembre 2012" (Decreto del Direttore Generale 82/2012, 2012), después de trece años.

\section{Comparación de ambas formaciones}

Hay diferencias sustanciales entre el sistema educativo español e italiano, sobre todo, en lo que concierne al acceso a la universidad y a la formación de profesorado.

Así, en España puede acceder a los estudios universitarios sólo quien haya realizado el bachillerato, o ciclos formativos de grado superior (CFGS), con limitación de plazas. No es así en Italia, donde cualquiera puede decidir ir a la universidad, independientemente de la educación secundaria postobligatoria que haya recibido.

También, según el sistema educativo español, hay que seguir criterios precisos de admisión a la Universidad, en cambio, en el sistema educativo italiano, no están establecidos, a menos que lo decida la universidad misma. En consecuencia, esta importante diferencia hace que, en Italia, cualquiera puede enseñar en una escuela pública o privada, sin haber superado nunca cualquier tipo de selectividad.

Además, en el Real Decreto 99/2011 (Real Decreto 99/2011, 2011: p. 6), se establecen los requisitos de admisión a un doctorado en España, entre los cuales hay que estar en posesión de al menos 60 créditos de nivel de Máster. En cambio, en Italia, conforme al "DM 224/1999” (Decreto Ministeriale 224/1999, 1999), únicamente es requisito la posesión de la licenciatura para acceder a la oposición para el Doctorado.

Actualmente, en Italia, se está promulgando un nueva reforma educativa que, en este momento, es un proyecto de ley (DDL) llamado "Buona Scuola" (Disegno di Legge "Buona Scuola” 2994, 2014), que ya causó la oposición de miles de docentes. Entre las novedades más importantes se señalan: la selección del director de su claustro de profesores, según un coloquio y sin tener en cuenta ninguna lista con las puntuaciones personales de cada docente (elaboradas considerando años de enseñanza en la escuelas, títulos otorgados, notas, etc.), y la eliminación de TFA o PAS, es decir de cursos de formación de profesorado, sustituyéndolos con la superación de una oposición como único camino para ser titulares.

\section{Posibles propuestas de mejora}

El estudio del sistema educativo de un país debe situarse en el contexto de los profundos cambios sociales y educativos que se han vivido en los últimos años. Es oportuno partir de una visión global que responda a las exigencias del contexto internacional, para diseñar unas pautas de intervención concretas y capaces de mejorar la calidad de la formación del profesorado.

En 2011, los informes de la Organización para la Cooperación y el Desarrollo Económicos (OCDE) sitúan 
a Italia y España en la cola de Europa, aunque sean potencias económicas mundiales. Sus resultados exponen la necesidad de cambios en la formación del profesorado y de criterios educativos homogéneos a nivel internacional. Esto no significa dejar de tomar en consideración el análisis del propio entorno escolar, es decir, tener en cuenta una planificación local de las ofertas y ofrecer una buena formación práctica según las exigencias de la realidad concreta en la que un docente va a enseñar, sino hacer converger los criterios de formación a nivel global $\mathrm{y}$, al mismo tiempo, tomar en consideración las necesidades específicas del propio territorio.

En 2011, la OCDE convocó la primera Cumbre Internacional sobre la Profesión Docente. Entre los temas tratados, destacan la formación de los docentes, el desarrollo profesional y la implicación del profesorado en la reforma de los sistemas educativos. Entre sus conclusiones principales, se pueden subrayar la necesidad de disponer de unos docentes de calidad a partir de los cambios escolares y del sistema educativo y la urgencia de políticas de profesorado que adopten un enfoque global (OECD, 2011).

A la luz de estas consideraciones, posibles propuestas de mejora implicarían un estudio de campo sobre la calidad de la formación de profesorado en España e Italia, comparando, también, qué país sigue criterios más homogéneos a los demás en la Unión Europea y cuál otorga más importancia a la formación práctica, equilibrando la enseñanza universitaria y la experiencia en los centros escolares. Además, un estudio más avanzado podría confrontar los efectos en el plano social y económico de los criterios seguidos en el camino de formación italiano y español.

\section{Conclusiones}

A menudo, las reformas educativas nacen asociadas a los cambios de legislatura. De hecho, cada vez que cambia el sistema político de un gobierno, parece producirse una nueva ley de educación, sin tener en cuenta los cambios educativos de fondo que deberían producirse. El profesorado, su preparación y metodología docente, deben adaptarse a los tiempos, así como a las necesidades de la sociedad. Es el momento de homologar los criterios formativos a nivel internacional y global, pero de acuerdo con la propia realidad local. El poder para hacerlo lo tienen las personas, más que las legislaciones, los profesores deben formarse para estar preparados para el cambio y el aprendizaje continuo.

\section{Referencias}

Decreto del Direttore Generale 82/2012, de 24 de septiembre, Indizione dei concorsi a posti e cattedre, per titoli ed esami, finalizzati al reclutamento del personale docente nelle scuole dell'infanzia, primaria, secondaria di I e II grado. Gazzetta Ufficiale, 75, de 25 de septiembre de 2012. http://www.gazzettaufficiale.it/gunewsletter/dettaglio. jsp?service $=4 \&$ datagu $=2012-09-25 \&$ task $=$ dettaglio \&
numgu=75\&redaz=12E05411\&tmstp=134934541988 6

Decreto del Presidente della Repubblica 89/2009 de 20 de marzo, Revisione dell'assetto ordinamentale, organizzativo e didattico della scuola dell'infanzia e del primo ciclo di istruzione ai sensi dell'articolo 64, comma 4, del decreto-legge 25 giugno 2008, n. 112, convertito, con modificazioni, dalla legge 6 agosto 2008, n. 133. Gazzetta Ufficiale, 162, de 15 de julio de 2009.

http://www.normattiva.it/uri-res/N2Ls?urn:nir:stato:d ecreto.legge:2009-03-20;89

Decreto Ministeriale 224/1999, de 30 de abril, Regolamento in materia di dottorato di ricerca. Gazzetta Ufficiale, 224, de 30 de abril de 1999. http://www.miur.it/0006Menu_C/0012Docume/0098 Normat/2012Regola_cf2.htm

Decreto Ministeriale 249/2010, de 10 de septiembre, Regolamento concernente: "Definizione della disciplina dei requisiti e delle modalità della formazione iniziale degli insegnanti della scuola dell'infanzia, della scuola primaria e della scuola secondaria di primo e secondo grado, ai sensi dell'articolo 2, comma 416, della legge 24 dicembre 2007, n. 244». Gazzetta Ufficiale, 24, de 31 de enero de

2011. http://attiministeriali.miur.it/anno-2011/novembre/dm -11112011.aspx

Decreto Ministeriale 11 novembre 2011, de 11 de noviembre, Definizione delle modalità di svolgimento $e$ delle caratteristiche delle prove di accesso ai percorsi di tirocinio formativo attivo di cui all'articolo 15, comma 1, del decreto del Ministro dell'istruzione, dell'università e della ricerca 10 settembre 2010, n. 249. Gazzetta Ufficiale, 288, de 12 de diciembre de 2011. http://attiministeriali.miur.it/anno-2011/novembre/dm -11112011.aspx

Decreto Ministeriale 353/14, de 22 de mayo, Graduatorie di istituto -triennio 2014/2016Disposizioni in merito alla costituzione delle graduatorie di circolo e di istituto relative al personale docente ed educativo per gli anni scolastici 2014-2015, 2015-2016, 2016-2017. Ministero dell'Istruzione, dell'Università e della Ricerca, 353, de 22 de mayo de 2014. http://webcache.googleusercontent.com/search?q=cac he:r7eOPYwFxGEJ:hubmiur.pubblica.istruzione.it/P RTA-TitoliAccesso/documenti/DM_353_22_maggio_ 2014.pdf $+\& c d=6 \& h l=i t \& c t=$ lnk \&gl=it

Direttiva 1999/70/CE, de 28 de junio, relativa all'accordo quadro CES, UNICE e CEEP sul lavoro a tempo determinato. Gazzetta Ufficiale della Unione Europea, L175, de 10 de julio de 1999. http://eur-lex.europa.eu/LexUriServ/LexUriServ.do?u ri=CELEX:31999L0070:IT:HTML

Disegno di Legge "Buona Scuola", de 27 de marzo, Riforma del sistema nazionale di istruzione $e$ formazione e delega per il riordino delle disposizioni legislative vigenti. Ministero dell'Istruzione, dell’Università e della Ricerca, 2994, de 27 de marzo de 2014. 
http://www.camera.it/_dati/leg17/lavori/stampati/pdf/ 17PDL0029700.pdf

Legge 53/03, de 28 de marzo, Delega al Governo per la definizione delle norme generali sull'istruzione e dei livelli essenziali delle prestazioni in materia $d i$ istruzione e formazione professionale. Gazzetta Ufficiale, 77, de 2 de abril de 2003. http:/www.normattiva.it/uri-res/N2Ls?urn:nir:stato:le gge:2003;53

Legge 296/06, de 27 de diciembre, Disposizioni per la formazione del bilancio annuale e pluriennale dello Stato (legge finanziaria 2007). Gazzetta Ufficiale, 299, de 27 de diciembre de 2006. http://www.normattiva.it/uri-res/N2Ls?urn:nir:stato:le gge:2006-12-27;296

Legge 169/08, de 30 de octubre, Conversione in legge, con modificazioni, del decreto-legge $1^{\circ}$ settembre $2008, n .137$, recante disposizioni urgenti in materia di istruzione e università. Gazzetta Ufficiale, 169, de 31 de octubre de 2008. http://www.normattiva.it/uri-res/N2Ls?urn:nir:stato:le gge:2008;169

Ley Orgánica 1/1990, de 3 de octubre, de Ordenación General del Sistema Educativo. Boletín Oficial del Estado, 238, de 4 de octubre de 1990. http://www.boe.es/buscar/doc.php?id=BOE-A-1990-2 4172

Ley Orgánica 2/2006, de 3 de mayo, de Educación. Boletín Oficial del Estado, 35, de 4 de mayo de 2006. http://www.boe.es/buscar/doc.php?id=BOE-A-2006-7 899

Ley Orgánica 8/2013, de 9 de diciembre, para la mejora de la calidad educativa. Boletín Oficial del Estado, 295, de 10 de diciembre de 2013. https://www.boe.es/diario_boe/txt.php?id=BOE-A-20 13-12886

Ministerio de Educación, Cultura y Deporte (Sin fecha). http://www.mecd.gob.es/educacion-mecd/areas-educa cion/sistema-educativo/lomce.html

OECD (2011). Building a High-Quality Teaching Profession. Lessons from around the World. Paris, OECD.

http://www2.ed.gov/about/inits/ed/internationaled/tea ching-summit-2011.html
Orden del Ministerio de Educación y Ciencia ECI/3858/2007, de 27 de diciembre, por la que se establecen los requisitos para la verificación de los titulos universitarios oficiales que habiliten para el ejercicio de las profesiones de Profesor de Educación Secundaria Obligatoria y Bachillerato, Formación Profesional y Enseñanzas de Idiomas. Boletín Oficial del Estado, 312, de 29 de diciembre de 2007. http://www.boe.es/diario_boe/txt.php?id=BOE-A-200 7-22450

Real Decreto 276/2007, de 23 de febrero, por el que se aprueba el Reglamento de ingreso, accesos $y$ adquisición de nuevas especialidades en los cuerpos docentes a que se refiere la Ley Orgánica 2/2006, de 3 de mayo, de Educación, y se regula el régimen transitorio de ingreso a que se refiere la disposición transitoria decimoséptima de la citada ley. Boletín Oficial del Estado, 53, de 2 de marzo de 2007. http://www.boe.es/buscar/act.php?id=BOE-A-2007-4 372

Real Decreto 1393/2007, de 29 de octubre, por el que se establece la ordenación de las enseñanzas universitarias oficiales. Boletín Oficial del Estado, 260, de 30 de octubre de 2007. http://www.boe.es/buscar/act.php?id=BOE-A-2007-1 8770

Real Decreto 1892/2008, de 14 de noviembre, por el que se regulan las condiciones para el acceso a las enseñanzas universitarias oficiales de grado y los procedimientos de admisión a las universidades públicas españolas. Boletín Oficial del Estado, 283, de 24 de noviembre de 2008. http://www.boe.es/diario_boe/txt.php?id=BOE-A-200 8-18947

Real Decreto 99/2011, de 28 de enero, por el que se regulan las enseñanzas oficiales de doctorado. Boletín Oficial del Estado, 35, 10 de febrero de 2011. http://www.boe.es/buscar/doc.php?id=BOE-A-2011-2 541

Tiana Ferrer, A. (2012). Los motivos de las leyes. Escuela. 3.957, 3 http://www.colectivolorenzoluzuriaga.com/PDF/Octu bre\%202012.pdf 